\title{
MJN NURSES' AWARENESS AND PERCEPTION OF FOUR SCORE SCALE VERSUS GLASGOW COMA SCALE TOOL USED AMONG INTUBATED PATIENTS
}

\author{
Mara Ross Abrina Yglesias, ${ }^{1}$ Emiliano lan B. Suson II \\ ${ }^{1}$ Vicente Sotto Memorial Medical Center, Philippines \\ ${ }^{2}$ Cebu Normal University, Philippines \\ *Corresponding Author’s Email: susonei@cnu.edu.ph
}

\begin{abstract}
Level of consciousness has always been part of the endorsement and/or data in the nurses notes. Commonly used in the practice is still the GCS. It has also been an observation that in different cases, even though the patients are awake, since they are intubated, verbal response is immediately scored as 1 which contributes to a low GCS score that is relative to a bad prognosis. Also, assessment for complex reflexes are left out which can entail a better assessment on LOC. Hence, this study aims to compare the assessment, awareness, and perception of nurses in different levels of expertise assigned in the emergency department on the level of consciousness of intubated patients using the FSS and GCS tools to hasten triage categorization. It utilized a quantitative, descriptive-comparative type of research conducted in a government hospital in Cebu City. Specifically, on the areas of Emergency Department and Non- Trauma, where the researcher has easier access to registered nurses who are taking care of intubated patients. The researcher utilized awareness and perception tools and the FSS and GCS where researcher used complete enumeration involving 32 nurses. In light with the findings of this study, this can be utilized through thorough introduction of the FSS by an expert and on how to utilize the tool.
\end{abstract}

Keywords: Level of consciousness, Glasgow Coma Scale, Full Outline of Unresponsiveness Score, Intubated Patients

\section{INTRODUCTION \\ "Control of consciousness determines the quality oflife (Csikszentmihalyi, 1990)."}

Every person act on his own freewill, mind, and consciousness. He has control over his speech and actions. Hence, determines the quality of his life. From the traditional temperature, pulse, respiration, blood pressure and pain score taken during assessment, the level of consciousness (LOC) are already usually included upon initial assessment and is continuously monitored for any changes all throughout. These vital signs are taken as baseline data to later check for any fluctuations, improvement or regression in the condition of patients.

Within the spectrum of consciousness, scoring systems have been developed to obtain a fast- comprehensive assessment of coma to facilitate communication among healthcare personnel as well as to monitor changes for therapeutic decision and prognostic information. Scales have also been constructed to standardize examination of the unconscious patients. It also allows the grading of an unconscious patient over time which would indicate changes in clinical condition so that outcome may be predicted (Faruq, 2014).

The Glasgow Coma Scale (GCS) is the most widely used tool for the evaluation of the level of consciousness. However, there is a new tool that has been developed by a team of researchers led by Wijdicks, at the Mayo Clinic in Rochester, Minnesota. They have created an assessment tool called FOUR (Full Outline of Unresponsiveness) Score (FSS), which some facilities are using in place of, or in combination with the GCS (American Healthcare Education Services, 2016). 
This new score consists of four components, namely: eye, motor, brainstem and respiration, and each component has a maximum score of 4 . The FOUR score had a $20 \%$ higher predictive value for patients at risk for higher mortality for poor outcome than the GCS, according to a recent study's findings. The score is easy to learn, has very good inter- rater reliability, and provides a more accurate picture of the patient's status than the GCS (American Healthcare Education Services, 2016).

According to the researchers that developed the score, the FOUR score addresses some of the shortcomings of the GCS but may be more difficult to administer. The GCS fails to assess the verbal score in patients that are intubated and isn't able to test brainstem reflexes. In contrast, the FOUR scale does not need a verbal response, thus allowing complete testing in intubated patients, and gives information on brainstem activity and respiratory patterns. This additional information could give the team more insight into the need for intubation. Most importantly, the FOUR scale demonstrated a better discrimination between the good (recovery of independent living) and poor neurological status at 3 months as compared with the GCS (American Healthcare Education Services, 2016).

Level of consciousness has always been part of the endorsement and/or data in the nurse's notes. Commonly used in the practice is still the GCS. It has also been an observation that in different cases, even though the patients are awake, since they are intubated, verbal response is immediately scored as 1 which contributes to a low GCS score that is relative to a bad prognosis. Also, assessment for complex reflexes are left out which can entail a better assessment on LOC.

In the past 30 years, many coma scales have been proposed to use as an alternative to GCS, but none with success. Not all change is an improvement, but there can be no improvement without change, and the validity of the new scale first needs to be corroborated when used in a general hospital setting be examiners other than neuroscience professionals (Laureys, Ledoux \& Piret 2005).

Up until 2005, when the FOUR Score Scale was then published by Mayo Clinic, several studies have been done comparing it to GCS. Mainly, the studies are done in Intensive Care Units (ICU) and made no mention of Emergency Departments (Faruq, 2014). Most studies are done in the United States, Iran, and India but not yet in the Philippines. Hence, this study aims to compare the assessment, awareness, and perception of nurses in different levels of expertise assigned in the emergency department on the level of consciousness of intubated patients using the FSS and GCS tools to hasten triage categorization.

\section{Conceptual Framework}

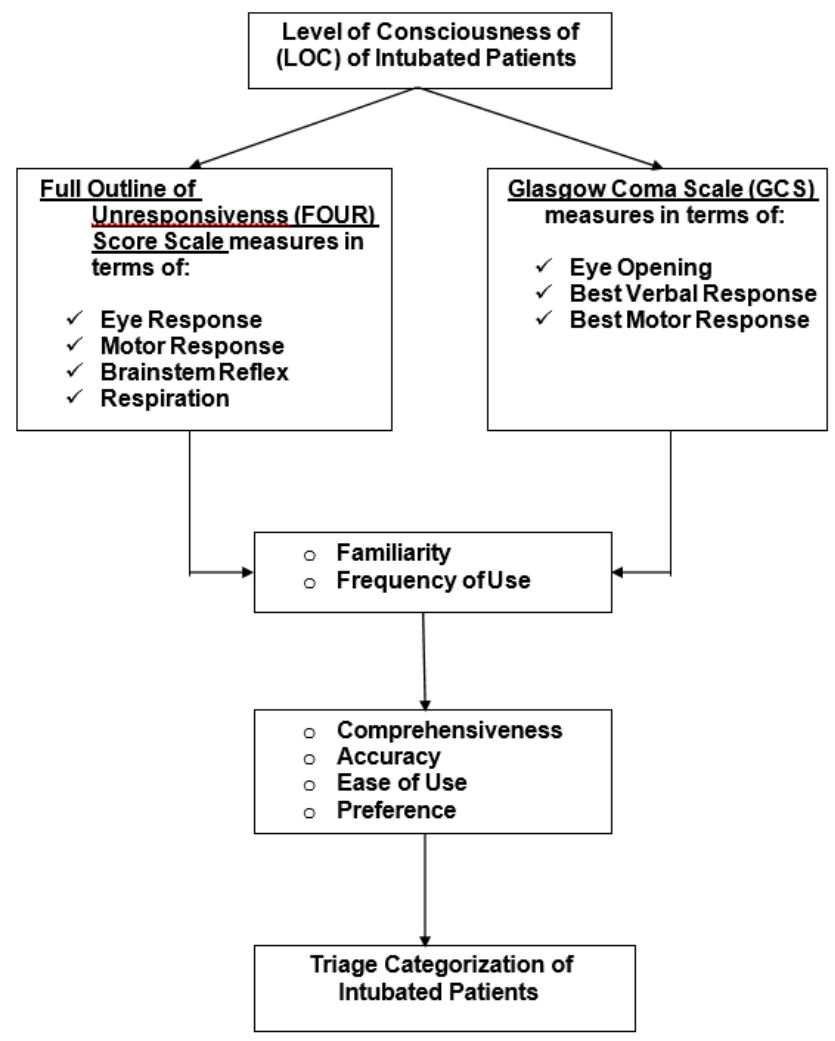

Figure 1: Conceptual Framework of the Study

The study seeks to provide an elaborate comparison of the FOUR Score Scale and Glasgow Coma Scale. In light of the widely used GCS tool in the practice, FSS is a newly developed and introduced tool to promote better assessment and hasten triage categorization of intubated patients suffering from trauma, stroke, thyrotoxic crisis, asthma in acute exacerbation, and the like.

An ideal coma scale should be reliable, valid, easy to use, easy to remember and of course an indicator of patient outcome. Raters who examine patients should be able to test accuracy of an ideal coma scale. Such scales should not involve additional cards or tools and should be useful in variety of patients with acute neurological disease not exclusively traumatic brain injury. Medical intervention like endotracheal intubation should not make assessment of certain components unreliable. There should not be any scope for educated guess or pseudo scoring in an ideal coma scale and it should be 
easy to memorize all components of the scale. The scale should have internal consistency which means when component changes parallel changes should be seen in other components. Lower scores in an ideal scale should indicate higher chances in mortality or future disability in a patient. Above all, an ideal coma scale should not be too simple or too complicated (Faruq, 2014). The Glasgow Coma Scale has been commonly used in the practice since its development in 1974 (GCS Eye Verbal Motor 2019). It has been utilized with familiarity and frequency. On 2005, the researchers at Mayo Clinic led by Dr. Wijdicks developed the Full Outline of Unresponsiveness Score Scale (Danielson et al., 2009).

The researcher compares both the tools in terms of comprehensiveness, accuracy, ease of use, and preference through nurses' exposure to the tools by using them in assessing the level of consciousness. In which they will be able to determine which suits best as an assessment tool in assessing LOC of intubated patients to hasten triage categorization.

\section{Problem Statement}

This study aims to compare nurses' exposure to two kinds of scale, FSS and GCS in assessing LOC among intubated patients. It specifically seeks to explain the following:

1. What is the level of awareness among nurses regarding FSS and GCS in terms of

- familiarity; and

- frequency of use

2. What are the nurses' exposure on FSS and GCS as assessment tool in terms of

- comprehensiveness;

- accuracy;

- ease of use; and

- preference

\section{Significance of the Study}

The study will pave way for innovative, comprehensive and distinct way of assessing LOC of intubated patients. This study will be of great benefit to the following:

Nurses. This study will give nurses an easier, more accurate and comprehensive way of assessing the level of consciousness in intubated patients. They will also be able to monitor subtle changes in level of consciousness that may need urgent actions.

Nursing Practice. This study will provide nursing practice a new body of knowledge which will pave a new light on how to assess level of consciousness among intubated patients.

Nursing Students and Researchers. The student nurses will be able to expand their knowledge and assessment skills as FOUR Score Scale being a new tool can be used in intubated patients and will not only be limited to the use of Glasgow Coma Scale.

Health Professionals. The health professional will be able to understand the condition of patients in an easier manner and to what extent are their needed care.

Future Researchers. This research can provide basis and reference for future studies about assessment of level of consciousness in intubated patients and in comparison, with other existing tools.

\section{Review of Related Literature}

The deliberative nursing process developed by Ida Jean Orlando allows nurses to formulate an effective nursing care plan that can easily be adapted when and if any complexity come up with the patient. She proposed that patients have their own meanings and interpretations of situations and therefore nurses must validate their interferences and analyzes with patients before drawing conclusions (Alligood \& Tomey, 2013).

Alligood \& Tomey (2013) also mentioned that the theory explains that the role of the nurse is to find out and meet the immediate needs of patients for help, the nature of their distress and provide the help they needed. With this context, assessment as the initial step in the nursing process plays an essential role in determining what actions or interventions to plan in order to address these needs.

Nurses have traditionally relied on five vital signs to initially assess their patients: temperature, pulse, blood pressure, respiratory rate and oxygen saturation (Ahrens, 2008). For over 100 years, nurses have performed this surveillance using the same vital signs and prompt detection and reporting of changes in these vital signs are essential as delays in initiating appropriate treatment can detrimentally affect the patient's outcome (Chalfin et al., 2007).

However, as patients hospitalized today are sicker 
than in the past, as they have more complex health problems and are far more likely to become seriously ill during their admission, these vital signs may not be adequate to identify those who are clinically deteriorating. In addition, patients who were once too sick to be operated on are now undergoing complex surgical procedures. This, coupled with the increasing demand for beds, means that ward nurses are often caring for patients who previously would have been cared for in a high-dependency or intensive care unit. Furthermore, system factors such as skill mix, nurse: patient ratios and bed shortages significantly impact on the quality of nursing care delivered in these environments (Coventry \& Elliot, 2012).

This challenging situation is further complicated by increasing patient survival rates, which have resulted in an increasingly complex and older patient population (James et al., 2010). Patients aged 65 and older, for example, have twice the risk of younger adults of developing peri-operative complications. They are also more likely to be admitted as emergencies and undergo emergency surgery. Diminished reserves in cognitive, renal and hepatic function also contribute to older patients being a group at high risk of adverse events. As such, the five traditional vital signs may not be adequate to detect clinical changes in patients who have more complex care needs than nurses have encountered in the past(Thornlow, 2009).

Before an acute change in a patient's physiology can be recognized, the vital signs must be assessed. In addition to the traditional temperature, pulse, blood pressure, respiratory rate and oxygen saturation, pain, level of consciousness, and urine output are added in the study, Critical Care: The Eight Vital Signs of Patient Monitoring by Coventry \& Elliott (2012).

As many factors can alter a patient's level of consciousness, nurses should assess it routinely along with other vital signs. Cognitive deficits are often subtle in their presentation and can easily be overlooked by nurses who are focused on more obvious physical problems, such as severe pain. Unfortunately, many nurses do not have a good understanding of the underlying mechanisms that produce altered levels of consciousness. For example, subtle changes in a patient's personality such as a patient who is uncharacteristically abrupt or aggressive could suggest alcohol withdrawal, hypoxia, hypercapnia, hypoglycemia, hypotension or a medication side effect of benzodiazepines, anxiolytics, opioids, etc. As such, nurses must always be alert for subtle changes with their parents which warrant further investigation (Coventry \& Elliot, 2012).

In 1974 the Institute of Neurological Sciences, Glasgow, was a world leader in brain injury research and clinical care. Professor Jennett and Mr Teasdale, (at that time a neurosurgical senior registrar), published a paper in the Lancet on the Assessment of Coma and Impaired Consciousness that proposed a structured method of assessment that would become known as the Glasgow Coma Scale (GCS Eyes Verbal Motor 2019).

The Glasgow Coma Scale (GCS) has been the gold standard of neurologic assessment for trauma patients since its development by Jennett and Teasdale in the early 1970s. The GCS was found to be a simple tool to use. It became the method of choice for trauma care practitioners to document neurologic findings over time and predict functional outcome. Although the scale has been shown to be effective, many authors have cited weaknesses in the scale including the inability to predict outcome, variation in inter-rater reliability, and the inconsistent use by caregivers in the prehospital and hospital settings (Fischer \& Mathieson, 2001).

Looking into the history and evolution of coma scale, it appears that GCS so far stood the test of time for 30 years since its introduction until 2005 when it was challenged by the proponents of FSS. In spite of its draw backs GCS is still being used by clinicians of many institutions because of its simplicity of use. But it has lost its usefulness in severe neuro impaired patients more so in the settings of ICUs. At best we can conclude that GCS is probably more suitable for simpler nonintubated patients without brainstem dysfunction. It is when the FSS has come to light in 2005 developed by the Mayo Clinic. It has the potential to achieve widespread acceptance among our physician community to become a universally acceptable gold standard Coma Scale due to its capacity to provide greater neurologic information by quantifying neurologic consciousness through eye and motor responses, brainstem reflexes and breathing pattern (Faruq, 2014).

According also to Faruq (2014), as a result probability of in hospital mortality is higher for the lowest total FOUR score when compared with that of GCS. FOUR score has been subjected to validation studies11-16 in different scenarios like neuro ICU, emergency departments, medical ICU, comatose stroke patients in acute stroke unit, traumatic brain injuries etc. It has been compared with GCS in these validation studies and excellent inter- rater agreements have been 
observed (Faruq, 2014).

The Full Outline of Unresponsiveness is a newly designed and clinically tested tool that assesses patients with impaired level of consciousness. It was developed in the Neurocritical Care Unit at the Mayo Clinic in Rochester, Minnesota (Danielson et al., 2009).

There are four parameters evaluated namely: eye response, motor response, brainstem reflex and respiration which involves breathing pattern and ventilation. The eye response is scored as 4: Eyelids open and tracking, or blinking on command; 3: Eyelids open but not tracking; 2: Eyelids closed but open to loud voice; 1: Eyelids closed but open to pain and; 0: eyelids closed with pain. The motor response is scored as 4: Makes signs (thumbs up, fist, other); 3: Localizing to pain; 2: Flexion response to pain; 1: No response to pain and; 0: Generalized myoclonus status. The brainstem reflex is scored as 4: Pupil reflexes present, corneal reflexes and cough present; 3: One pupil wide and fixed, corneal reflexes present and cough present; 2: Pupil reflexes absent and corneal reflexes present or 2: Pupil reflexes present and corneal reflexes absent; 1: Pupil reflexes absent, corneal reflexes absent, cough present and; 0 : Pupil reflexes absent, corneal reflexes absent, cough absent. While the respiration is scored as 4: Not intubated with regular breathing; 3: Not intubated with CheyneStokes type of breathing; 2: Not intubated with irregular breathing; 1: Intubated with breathing above ventilator rate; and 0 : Intubated with breathing at ventilator rate. The overall result is obtained by summing the scores assigned to each of the parameters. The minimum obtainable is 0 while the maximum is 16 . Outcomes suggest that the lower the score, the greater the coma gravity (Danielson et al., 2009).

The Glasgow Coma Scale is used to describe the general level of consciousness in patients and to define broad categories of head injury. The GCS is divided into three categories, Eye opening (E), Motor response (M), and Verbal response (V). The score is determined by the sum of the score of the three categories, with a maximum of 15 and a minimum score of 3 , whereas, GCS score $=E$ $+\mathrm{M}+\mathrm{V}$. The eye opening is scored as 4: Spontaneously; 3: To verbal command; 2 : To pain and; 1 : No response. The motor response is scored as 6: Obeys command; 5: Localizes pain; 4: Flexion withdrawal; 3: Flexion abnormal (decorticate); 2: Extension (decerebrate) and; 1: No response. While the verbal response is is scored as 5: Oriented and converses; 4: Disoriented and converses; 3: Inappropriate words; cries; 2: Incomprehensible sounds and; 1: No response. A GCS score of 8 is comatose and 3 is unresponsive (Christensen, 2014).

In the study of Comparison of Glasgow Coma Scale with Full Outline of Unresponsiveness Score in Measuring Consciousness Level of Endotracheal Tube Intubated Patient in the Intensive Care Unit, it has been discussed that good measuring scale of consciousness assessment is valid, reliable, and easy to use. This research obtained reliability value of GCS 0.718 and four score of 0.759 . This has made the researchers to conclude that FSS has better sensitivity and specificity than GCS. Further research is needed with different samples in different clinical settings (Oktarina \& Simajuntak, 2018).

In another study entitled, Comparison Between FOUR Score and GCS in Assessing Patients with Traumatic Head Injury: A Tertiary Centre Study, it has been discussed that head injuries are a major cause of mortality and morbidity across the world. Effective initial assessment and early intervention is of importance in patients with traumatic brain injury, so as to ensure the maximum favorable outcome. Glasgow Coma Scale is the widely accepted scale to assess severity in head injury patients, albeit with many inadequacies. The objective of this study was to test the validity of full outline of unresponsiveness score, an alternate tool, in assessing severity in patients with traumatic brain injury. As per the study results of this study, GCS and FOUR scores show comparable results in the assessment of patients with Traumatic Brain Injury. There is excellent statistical correlation between the two scoring systems. Additionally, FOUR score furnishes better details regarding the neurological status of the patient. The FOUR score can be applied as an effective reference to evaluate consciousness status in management of head injury. It can be a strong ally for the clinician in detecting and stratifying patients with severe head injuries and also in monitoring efficacy of treatment. With further clinical research, this tool can supersede GCS as the monitoring tool of choice in head injury (Nair et al., 2017).

Another study was the Validity of the FOUR Score Coma Scale in the Medical Intensive Care Unit by Danielson et al. (2009) that evaluated the validity of the FOUR score when used by the staff members in the medical ICU. The study evaluated 100 critically ill patients from May 1, 2007 to April 30, 2008 and for each patient, the FOUR score and GCS were determined by a randomly selected staff pair such as nurse/fellow, 
nurse/consultant, fellow/fellow, or fellow/consultant (Danielson et al., 2009).

Although the GCS has been widely used in hospital settings and is considered a standard assessment tool, it has several shortcomings. The usefulness of a verbal component in assessing level of consciousness can be questioned. First, the verbal component of the GCS tests primarily orientation, which quickly becomes abnormal in agitated and confused patients without impaired consciousness. Conversely, many patients with little or no verbal response are alert. Moreover, the verbal response component of the GCS cannot be assessed in critically ill patients who have undergone intubation; in fact, verbal response could not be reliably assessed in 45 of the 100 patients in the Validity of the FOUR Score Coma Scale in the Medical Intensive Care Unit study of Danielson et al. (2009). Second, and most importantly, the GCS does not assess brainstem reflexes, eye movements, or complex motor responses in patients with altered consciousness (reliability). Furthermore, the GCS score is numerically skewed toward motor responses (linearity) (Danielson et al., 2009).

Most of the studies regarding FOUR Score were focused on intensive care units as most patients are expected to be intubated. However, due to being new, no reports or study has been made yet regarding its utilization in local hospital settings. It has also made no mention in emergency departments where most of the cases are urgent and emergent where patients suffering from stroke, heart attack, thyrotoxic crisis, and asthma in acute exacerbation are initially rushed, intubated, and closely monitored for any alteration in consciousness.

\section{METHODOLOGY}

\section{Research Design}

This is a quantitative, descriptive-comparative type of research. This type of research aims to describe, document, and identify significant similarities and differences among variables of the study. Quantitative research is based on the measurement of quantity and amount. It is applicable to phenomena that can be expressed in terms of quantity. The major purpose of descriptive research is the description of the state of affairs as it exists at present. The researcher has no control over the variables, does not aim to generate changes and can only report and compare the similarities and differences to better understand them (Richardson, 2018).

\section{Research Locale}

This study was conducted in Vicente Sotto Memorial Medical Center (VSMMC) Emergency Department Non- Trauma, Cebu City, where the researcher has easier access to registered nurses who are taking care of intubated patients. VSMMC as a tertiary hospital, various cases are being referred to the institution which can be triaged from Red tags, Yellow tags, and Green tags patients and these may include intubated patients who would need urgent, immediate, or intermediate care.

\section{Research Respondents and Sampling Technique}

The researcher used complete enumeration involving 32 nurses who were rotated that time in the Emergency Department Non-Trauma who consented voluntarily to participate in the study in the period of one month. They were using functional team nursing, and each was equally given the chance to take care of an intubated patient depending on their specific roles during their shift (Hardy, 2017).

\section{Research Instrument}

The researcher utilized awareness and perception tools and the FSS and GCS. The awareness and perception tools were researcher made and has undergone pilot testing. First, the respondents answered the awareness tool regarding the FSS and GCS. Then, they next proceeded to utilize both the tools in assessing same intubated patient. The last part was to answer the perception tool based on their experience after using both the coma scales.

The awareness tool is composed of five items that will measure their familiarity of the tools. The perception tool is composed of two items. The first item measured the perception on the tools of the respondent based on their comprehensiveness, accuracy, ease of use, and preference. While the second item measured the respondent's recommendation of the tools. Pilot test was conducted using the awareness and perception tools. The respondents who took the pilot test were not included in the study.

\section{Data Gathering Procedure}

Data gathering was divided into three phases: preparatory, working and the presentation. In the preparatory phase, the researcher conducted a comprehensive review of different available and prospect literature and sources that best provided pertinent data and information on the subjects being investigated. Reliability test and validation of the instruments by pilot 
testing them were facilitated in order for the tool to be standardized. The researcher also secured a letter noted by the researcher's adviser and approved by the Dean of the Graduate School and Vice President of Academic Affairs. The letters were addressed to the Medical Center Chief of VSMMC which was then forwarded to VSMMC Training and Research Division. When the proposal was certified that it has been technically reviewed, it was then endorsed to REC for ethical review. Upon approval of the REC Chair, the researcher proceeded to the next step.

The second phase was the working phase. After the researcher identified the target population, the researcher personally collected the data. Before the study proper, the researcher inform the respondents regarding the planned study and its purpose by telling them verbally what the researcher must do. An informed consent was distributed to confirm their participation and cooperation in the conduct of the study. The researcher assured the respondents that the data collected is treated with utmost confidentiality. All gathering of data were conducted when the participants were already off duty or after their shift. Once the respondents signed the consent, the researcher then started the gathering of the data. Since it is a non-participant observation type of data collection, the researcher did not give any information and opinion regarding her preference and perception of both the tools. Thus, also avoiding Hawthorne effect (Shuttleworth, 2009).

The researcher let the respondents first take the Awareness Tool regarding the FSS and GCS. It was followed by utilizing the FSS and GCS tools in assessing the same intubated patients. The results of their assessment using both the tools were compared. After completing the assessment tools, the respondents have taken the Perception Tool regarding how they perceive both the tools based on comprehensiveness, accuracy, ease of use and preference. This was only one time gathering of data from the respondents.

The data gathered were coded for easy handling and statistical manipulations on the computer. Scores were also totaled for easy encoding and interpretation. After the data gathering, office editing, categorizing, coding and tabulating preparation, statistical treatment and analysis followed. The numerical codes were coded and analyzed in the Microsoft Excel. Statistical treatment was also done by the researcher with the help of a statistician.

After the analysis and interpretation of the gathered data, the researcher continued to the final phase, the presentation phase. In the presentation phase, the researcher presented to the panel of evaluators the vital processes and pertinent information from chapters one to five.

\section{Statistical Treatment of Data}

Statistical treatment was applied after the intensive collection of data. Frequency distribution, percentage, weighted mean, and standard deviation were used. The frequency distribution, percentage, weighted mean and standard deviation were used in measuring level of awareness among nurses in terms of familiarity and frequency of use, nurse's exposure to FSS and GCS in terms of comprehensiveness, accuracy, ease of use, and preference.

\section{Ethical Considerations}

The researcher has been granted the approval for implementation of the study by the VSMMC Research Ethics Committee (REC) through ERC Protocol No. VSMMC-REC-I-2018-019. It has been signed and approved by Dr. Maria Philina P. Villamor, REC Chairperson last May 30, 2018.

The researcher made sure that the respondents signed the consent form with complete understanding of what they are participating in. The researcher assured the respondents that throughout the study, their anonymity is preserved. During data gathering, the researcher has given the respondents ample time to answer each question without imparting any personal biased opinion regarding the tool used in the study. How the nurses scored the LOC of the intubated patients did not affect their care and monitoring rendered to their respective patients as the score obtained using both the FSS and GCS were only used in comparing the tools only and were not used to predict any patient prognosis.

All information pertaining in the conduction and participation of the study were included in the informed consent read and signed by the respondents. Any questions, concerns, and clarifications by the respondents were addressed by the researcher prior, during, and after the study was conducted. The information collected is kept safely locked up by the researcher where she only has access to. The information is only known to the researcher, the Ethics Committee, and the Regulatory authority. Should in any case, the respondents chose to refuse or withdraw in the study, it is respected and not contradicted and was documented properly. However, in the entire course of the study, there was no withdrawal made from any of the respondents. 


\section{RESULTS AND DISCUSSION}

This chapter presents and discusses the findings based on the data gathered in relation to the specific problems of the study. Also, tables with their corresponding descriptions and interpretations are included here.

After the 30 days of collecting data in Emergency Department Non-Trauma, it yielded the following results:

Table 1: Nurses' Level of Awareness and Exposure to FSS and GCS

\begin{tabular}{|l|l|c|c|c|}
\hline \multirow{2}{*}{} & \multicolumn{3}{|c|}{ FSS } & \multicolumn{2}{c|}{ GCS } \\
\hline Variables & Mean & SD & Mean & SD \\
\hline Familiarity & 1.94 & 1.13 & 4.38 & 0.55 \\
\hline Frequency of Use & 1.78 & 1.39 & 4.91 & 0.53 \\
\hline Comprehensiveness & 5.44 & 2.23 & 7.90 & 2.16 \\
\hline Accuracy & 6.09 & 2.88 & 7.86 & 1.79 \\
\hline Ease of Use & 4.69 & 2.80 & 8.31 & 1.82 \\
\hline Preference & 4.44 & 2.65 & 8.53 & 1.83 \\
\hline
\end{tabular}

The nurses of the Emergency Department NonTrauma are more aware of the Glasgow Coma Scale than the FOUR Score Scale. It can be observed that the weighted mean values of their awareness of the tools are of extremes based on their familiarity of the tools which is then supported by their frequency of use of the tools. They are more familiar and frequent users of the GCS. Their mean value in familiarity and frequency of use in GCS is high and their standard deviation is low which means that their scores are close to each other. As the institution commonly use GCS, they are more accustomed to it than in the use of FSS. It can also be implied that most of the nurses heard of the FSS at first hand during the conduct of the study as shown in their low mean value in familiarity and frequency of use and closely spread standard deviation. We can infer that frequency of use of the tools is directly proportional to their familiarity of the tools. (Ramazani \& Hosseini, 2019).

Moreover, the nurses still perceive the GCS as more comprehensive, more accurate, easier to use and more preferable than the FSS. However, in the comprehensiveness and accuracy aspects, it can also be implied that the nurses can also find the FSS as a comprehensive and an accurate tool in assessing the level of consciousness of intubated patients as their weighted mean values based are not far behind from the GCS weighted mean value. While in the ease of use and preference aspects, the GCS is really on higher averages. This can imply that the nurses kept on using this tool for the longest time, are very familiar with and more comfortable with the tool. Consequently, it can also be concluded that they are resistant in using a new and/or unfamiliar tool. Also, due to the demanding workload of the Emergency Department, in order to save time and avoid errors and overlooking of workloads and other essential assessments, they would give more preference to the tool which gives them the ease of use. From the low value of the standard deviation of their scores which were closely spread, it can also be implied that comprehensiveness, accuracy, ease of use, and preference is directly proportional to their exposure to FSS and GCS.

\section{Comparison of FSS and GCS}

\begin{tabular}{|l|c|c|}
\hline & FSS & GCS \\
\hline Author & $\begin{array}{c}\text { Dr. Eelco Wijdicks and } \\
\text { colleagues in Mayo Clinic }\end{array}$ & $\begin{array}{c}\text { Dr. Graham Teasdale and } \\
\text { Prof. Bryan Jennett }\end{array}$ \\
\hline Year & 2005 & 1974 \\
\hline Place & Mayo Clinic & $\begin{array}{c}\text { Institute of Neurological } \\
\text { Sciences }\end{array}$ \\
\hline Target Assessment & LOC & LOC \\
\hline Specificity & $\begin{array}{c}\text { Eye Response Motor } \\
\text { Response Brain Reflexes }\end{array}$ & $\begin{array}{c}\text { Eye Response Motor } \\
\text { Response Verbal }\end{array}$ \\
\hline $\begin{array}{l}\text { Best Possible } \\
\text { Score }\end{array}$ & 16 & 15 \\
\hline $\begin{array}{l}\text { Lowest Possible } \\
\text { Score }\end{array}$ & 0 & 3 \\
\hline
\end{tabular}

The FOUR Score Scale was developed by the researchers of Mayo Clinic led by Dr. Eelco Wijdicks on 2005 at Mayo Clinic, Rochester, Minnesota. It consists of four components namely: eye, motor, brainstem and respiration. Each has a maximum score of 4 and lowest score of 0 . The score is easy to learn, has a very good interrater reliability, and provides a more accurate picture of the patient's status than the GCS. It assesses the brainstem reflex and respiratory patterns hence does not need a verbal response. This additional information gives more insight in the neurological status of the patients (American Healthcare, 2016).

On the other hand, Glasgow Coma Scale was developed by Dr. Graham Teasdale and Prof. Bryan Jennett in 1974 at the Institute of Neurological Sciences, Glasgow. With Dr. Teasdale's long interest in head injuries he joined the world class team led Professor Bryan Jennett. Prof. Jennett was a professor of neurosurgery and Dean of Medicine in at Glasgow 
University. The output of the "Glasgow School" revolutionized the management of head injured patients worldwide, hence the Glasgow Coma Scale. Due to the scale's simplicity and ease of communication, it was warmly welcomed in departments caring for patients with acute brain injury from trauma and other causes. Nurses rapidly welcomed the clarity of capture of important trends in the condition of a patient. Forty years after the original description, a review in The Lancet Neurology $(2014 ; 13: 844$ - 54) reported that the GCS was in use by neurosurgeons and other disciplines in more than 80 countries worldwide and had been translated into the national language in 74\% (GCS Eye Verbal Motor 2019).

The GCS has been continuously used in the practice frequently due to its familiarity. The FSS being new, has garnered different views from the nurses who participated in the study. One of which is, "Nindot ang FSS, lisod lang." They find the FSS of good quality, however, is hard to use. Another is, "Nindot and FSS, resistant to change mi, so stick to GCS gihapon mi." They also find FSS of good quality however they are resistant to change so they still stick to using the GCS. Another general view from the nurse's is' "Ganahan mi sa FSS pero mas dali gamiton gihapon gamiton ang GCS." They would prefer FSS however they still find the GCS easier to use.

Any alternative that would make work more effective and efficient would be highly beneficial. However, welcoming change such as a new tool in the area that the utilization may take longer than usual, staff may become resistant to change. Nevertheless, frequent exposure to practice of the new tool, nurses can become accustomed to it and can openly incorporate use of FSS in their practice as a new tool in assessing LOC of intubated patients.

Moreover, in order to impart new knowledge and improve nursing practice, one shall not stick to orthodox practice but be open to limitless possibility. As the nursing practice advances, continuous research must go together with nursing education as whether a better tool than the GCS used in the current practice can still be applicable. Ample time of learning in utilizing the FSS must also be given to the staff nurses.

As a patient advocate, whatever serves best must be given to the patient. As this new knowledge is a product of research and has undergone thorough evaluation, it is evidence-based and can be ethically accepted.

\section{CONCLUSION}

This chapter presents the conclusions drawn from the findings of the study and the recommendations that the researcher have come up for other researchers with the same interest.

In light of the findings of the study, it is concluded that the nurses of Vicente Sotto Memorial Medical Center Emergency Department Non-Trauma, acknowledged that apart from the Glasgow Coma Scale that has been used in assessing level of consciousness on intubated patients, there is also the Full Outline of Unresponsiveness Score Scale. The nurses are more familiar and more frequent users of the GCS than the FSS. They find the GCS highly comprehensive, highly accurate, easy to use, and more preferable. On the other hand, they find the FSS comprehensive, accurate, hard to use, and less preferable

\section{Recommendations}

After a thorough analysis of the data, the researcher made the following recommendations:

1. A similar study in the future to be conducted to other healthcare allies especially to the doctors if they find FSS more feasible than the GCS, more healthcare providers can recommend its utilization in the institution and even in the Philippine practice.

2. A thorough introduction of the FSS by an expert to the participants prior to the conduct of the study.

3. A wider scope of healthcare workers with varied levels of expertise (e.g. nurses: novice, advance beginner, competent and, expert) and their levels to be specified in the treatment of the data to determine how closely similar they utilize the tool.

4. A future study determining the inter-raters score of nurses and other health professionals in using the FSS.

5. A future study to look into patient outcome and prognosis with the use of the FSS.

6. A future comparative study of FSS with other LOC tools.

\section{Conflict of Interests}

The authors declare that they have no conflict of interest.

\section{ACKNOWLEDGEMENT}

The authors are thankful to the institutional authority for completion of the work. 


\section{REFERENCES}

Ahrens T. (2008). The most important vital signs are not being measured. Australian critical care: official journal of the Confederation of Australian Critical Care Nurses, 21(1), pp 3-5.

Alligood, M.R. \& Tomey, A.M. (2013). Nursing Theorists and their Work. Elsevier, USA.

American Healthcare Education Services (2016). Tips to treasure: the four score for neuro assessments. Retrieved from: https://www.rn.com/Pages/ResourceDetails.aspx?id=3024

Chalfin, D.B., Trzeciak, S., Likourezos, A., Baumann, B.M., Dellinger, R.P. \& DELAY-ED study group. (2007). Impact of Delayed Transfer of Critically Ill Patients from the Emergency Department to the Intensive Care Unit. Critical Care Medicine, 35(6), pp 1477-1483.

Christensen, B. (2014). Adult glasgow coma scale. Retrieved from https://emedicine.medscape.com/article/2172603overview

Coventry, A. \& Elliot, M. (2012). Critical care: the eight vital signs of patient monitoring. British Journal of Nursing, 21(10), pp 621-625.

Csikszentmihalyi, M. (1990). Flow: the psychology of optimal experience. Cambridge University Press, UK. Retrieved from: https://www.goodreads.com/quotes/tag/consciousness

Danielson, R.D., Elmer, J.L., Iyer, V.N., Mandrekar, J.N., Wijdicks, E.F. \& Zubkov, A.Y. (2009). Validity of the Four Score Coma Scale in the Medical Intensive Care Unit. Mayo Clinic proceedings, 84(8), pp 694-701.

Faruq, M.O. (2014). Looking for an ideal coma scale: It is time to replace GCS. Bangladesh Critical Care Journal, 2(1), pp 1-3.

Fischer, J. \& Mathieson, C. (2001). The History of Glasgow Coma Scale: Implications for Practice. Critical Care Nursing, 23(4), pp 52-8.

GCS Eyes Verbal Motor (2019). Who we are. The glasgow structured approach to assessment of the glasgow coma scale. Retrieved from: https://www.glasgowcomascale.org/who-we-are/

Hardy, L. (2017). The Advantages \& Disadvantages of Functional Nursing. pocketsense.com. Retrieved from: https://pocketsense.com/the-advantages-disadvantages-of-functional-nursing-12582245.html

James, J., Butler-Williams, C., Hunt, J. \& Cox, H. (2010). Vital signs for vital people: an exploratory study into the role of the healthcare assistant in recognizing, recording, and responding to the acutely ill patient in the general ward setting. Journal of nursing management, 18(5), pp 548-55.

Laureys, S., Ledoux, D. \& Piret, S. (2005). Quantifying consciousness. Lancet Neurology, 4, pp 789-90.

Nair, S.S., Surendran, A., Prabhakar, R.B. \& Chisti, M.M., (2017). Comparison Between Four Score and GCS In Assessing Patients with Traumatic Head Injury: A Tertiary Centre Study, International Surgery Journal, 4(2), pp 656-662.

Oktarina, Y. \& Simajuntak, C. (2018). Comparison of Glasgow Coma Scale with Full Outline of Unresponsiveness Score in Measuring Consciousness Level of Endotracheal Tube Intubated Patient in the Intensive Care Unit. International Journal of Research in Medical Sciences, 6(6), pp 1882- 1885.

Ramazani, J. \& Hosseini, M. (2019). Comparison of full outline of unresponsiveness score and Glasgow Coma Scale in Medical Intensive Care Unit. Annals of Cardiac Anaesthesia, 22(2), pp 143-148.

Richardson, H. (2018). Characteristics of a comparative research design. Retrieved from: https://classroom. synonym.com/characteristics-comparative-research design-8274567.html

Shuttleworth, M. (2009). Hawthorne effect. Retrieved from: https://explorable.com/hawthorne-effect

Thornlow, D. (2009). Increased risk for patient safety incidents in hospitalized older adults. Medsurg Nursing, 18(5) pp 287-91. 